\title{
Ultrasound of the chest and mediastinum in children, interventions and artefacts. WFUMB review paper (part 3)
}

\author{
Cheng Fang1, Joanna Jaworska ${ }^{2}$, Natalia Buda ${ }^{3}$, Ioana Mihaiela Ciuca ${ }^{4}$, Yi Dong ${ }^{5}$, Axel \\ Feldkamp $^{6}$, Jörg Jüngert ${ }^{7}$, Wojciech Kosiak ${ }^{8}$, Hans Joachim Mentzel ${ }^{9}$, Corina Pienar ${ }^{4}$, \\ Jorge S. Rabat ${ }^{10}$, Vasileios Rafailidis ${ }^{1}$, Simone Schrading ${ }^{11}$, Dagmar Schreiber-Dietrich ${ }^{12}$, \\ Christoph F Dietrich ${ }^{13,14}$
}

${ }^{1}$ Department of Radiology, King's College Hospital, London, United Kingdom, ${ }^{2}$ Institute of Mother and Child, Cystic Fibrosis Department, Warszawa, Poland, ${ }^{3}$ Internal Medicine, Connective Tissue Diseases and Geriatrics Department, Medical University of Gdansk, Poland, ${ }^{4}$ Department of Pediatrics, University of Medicine and Pharmacy "Victor Babes" Timisoara, Romania, ${ }^{5}$ Department of Ultrasound, Zhongshan Hospital, Fudan University, Shanghai, China, ${ }^{6}$ Pediatric Department, Sana Kliniken Duisburg GmbH, Germany, ${ }^{7}$ Department of Pediatrics and Adolescent Medicine, University Hospital Erlangen, Germany, ${ }^{8}$ Pediatric, Hematology and Oncology Department, Medical University of Gdansk, Poland, ${ }^{9}$ Section of Pediatric Radiology, Institute of Diagnostic and Interventional Radiology, University hospital Jena, Germany, ${ }^{10} \mathrm{Head}$ of Surgery Department. Universidad de Oriente. Ciudad Bolívar City. Bolivar State. Venezuela, ${ }^{11}$ Klinik für Radiologie und Nuklearmedizin, Luzerner Kantonsspital, Switzerland, ${ }^{12}$ Localinomed, Bern, Switzerland, ${ }^{13}$ Department Allgemeine Innere Medizin (DAIM), Kliniken Hirslanden Beau Site, Salem und Permanence, Bern, Switzerland, ${ }^{14}$ Sino-German Research Center of Ultrasound in Medicine, The First Affiliated Hospital of Zhengzhou University, Zhengzhou, China

\begin{abstract}
Ultrasound (US) is an ideal diagnostic tool for paediatric patients owning to its high spatial and temporal resolution, realtime imaging, and lack of ionizing radiation and bedside availability. The lack of superficial adipose tissue and favourable acoustic windows in children makes US the first line of investigation for evaluation of pleural and chest wall abnormalities.

In the first part of the topic the technical requirements were explained and the use of ultrasound in the lung and pleura in paediatric patients were discussed. In the second part lung parenchymal diseases with their subpleural consolidations are reflected. In the third part, the use of ultrasound for chest wall, mediastinum, diaphragmatic diseases, trachea, interventions and artifacts in paediatric patients are summarized.

Keywords: lung; chest; mediastinum; guideline; CEUS
\end{abstract}

In the first part of the "Lung ultrasound in children" topic the technical requirements were explained and the use of ultrasound (US) in the lung and pleura in paediatric patients were discussed [1]. In the second part, the

Received 05.03.2021 Accepted 21.05.2021

Med Ultrason

2022, Vol. 24, No 1, 65-76

Corresponding author: Prof. Dr. med. Christoph F. Dietrich, MBA

Department of Internal Medicine (DAIM)

Kliniken Hirslanden Bern, Beau Site,

Salem and Permanence

Schänzlihalde 11, 3031 Bern, Switzerland

E-mail: c.f.dietrich@googlemail.com lung parenchymal diseases with their subpleural consolidations were reflected [2].

Herewith, in the third part the use of US for chest wall, mediastinum, diaphragmatic diseases, trachea, interventions and artifacts in paediatric patients are summarized.

\section{CHEST WALL}

\section{Examination technique}

The superficially located structures of the thoracic wall close to the US transducer can be optimally examined with high-frequency linear probes (7-20 MHz). Ex- 
amining both sides of the thoracic wall is necessary to establish symmetry. The normal bone cortex appears as a uniformly hyperechoic structure with smooth margins, while costal cartilage is hypoechoic $[3,4]$.

Developmental abnormalities of the sternum include sternal tilt, pectus excavatum and carinatum $[3,5]$. Normal rib variants such as prominent anterior convex ribs, hypertrophic and/or wavy ribs, bifid ribs and asymmetric costochondral junctions may also be identified [3,5-7]. When evaluating a costochondral mass, the advantage of US over computed tomography (CT) or magnetic resonance imaging (MRI) is that it provides a more focused view [7]. Pathological findings should be imaged in two planes. Ribs are examined in their oblique presentation. In the current review breast examination is not included.

\section{Indication and pathological findings}

The main indication for chest wall US is a palpable mass in the thoracic region. Non-tender masses are usually benign, and US may provide a definitive diagnosis [6].

\section{Rib and clavicle fractures}

US is not the main imaging modality when evaluating rib or clavicle fractures, with radiography being the first line modality, but this is controversial. Radiographic signs may not be evident, when minor displacement occurs. In these cases, US examination may show disruption of the rib cortex (fracture lines, steps), haematoma or callous formation depending on the age of the fracture [5-7]. In addition, US is superior to plain radiographs when diagnosing traumatic dislodgement of costochondral cartilage or fractured sternum $[5,6]$. Documentation in two perpendicular axes is mandatory.

\section{Chest wall infections}

As sonographic findings precede radiographic abnormalities, US has an important role in assessing chest wall infections. US findings in cellulitis are diffusely increased echogenicity and thickness of the subcutaneous fat $[3,5,7]$. When cellulitis is complicated by a subcutaneous abscess, focal organised fluid collection with inflammatory changes in the surrounding fat can be seen on B-mode US and increased peripheral vascularity on colour Doppler [5,7,8]. Furthermore, US may facilitate drainage of the abscess by targeting the largest pocket of collection and used to follow-up chest wall infection, ensuring its resolution.

A normal-appearing costochondral cartilage on US in a patient presenting with costochondral pain may suggest costochondritis [3]. US signs of osteomyelitis include sub periosteal fluid collection, fluid in the proximity of the bone and bone cortex disruption $[3,5]$. While US diagnosis of osteomyelitis is feasible, rib osteomyelitis may be particularly difficult to diagnose with US alone and MRI is usually warranted.

\section{Benign chest wall tumours}

Benign solid masses of the chest wall are enumerated in Table I and figures 1-3 [3,6,8,9]. Lymph nodes are usually identified on US as hypoechoic solid structures with an echogenic fatty hilum with preserved architecture of blood vessels in the hilum $[3,6]$. In contrast, lipomas are well defined, echogenic masses, with minimal Doppler flow [6]. Desmoids are infiltrative hypoechoic, fibrillar tumours with muscular fascial involvement [9].

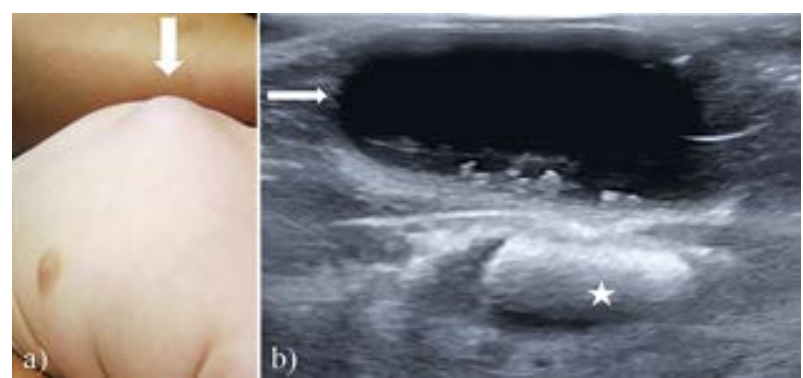

Fig 1. Clinical presentation: boy, 8 months, tumour above the sternum (a). Transverse scan: nearly anechoic tumour (arrow) above the sternum (star): epidermal cyst (b).

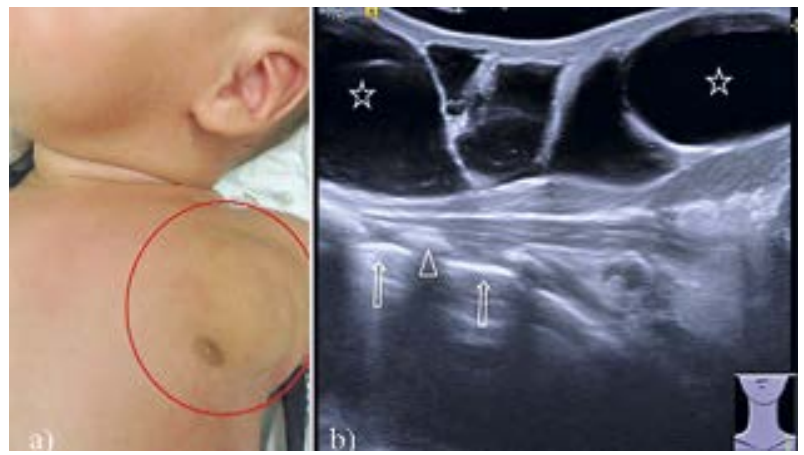

Fig 2. Three-month-old boy, asymptomatic superficial congenital tumour of the thorax and right axilla, lymphangioma. Clinical presentation (a). Ultrasound (b): macrocystic tumour with nearly anechoic cysts (stars) in the subcutaneous tissue respecting the integrity of the ventilated lung (white arrows) and the rib (white triangle).

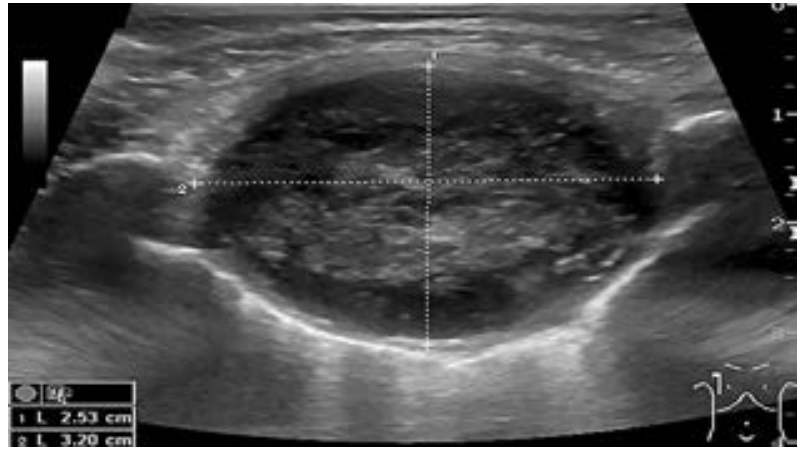

Fig 3. Tumour of the rib cartilage at the transition to the sternum: chondromyxoidfibroma. 
Table I. Chest wall tumours

\begin{tabular}{|c|c|c|}
\hline Type & Origin & \\
\hline \multirow[t]{12}{*}{ Benign } & Bone & Osteochondroma \\
\hline & & Osteoblastoma \\
\hline & & Osteoid osteoma \\
\hline & & Aneurysmal bone cyst \\
\hline & Subcutaneous tissue & Lymph nodes \\
\hline & & Lipoma/lipoblastoma \\
\hline & & Neurofibroma \\
\hline & & $\begin{array}{l}\text { Fibroma/desmoid } \\
\text { Epidermal cyst }\end{array}$ \\
\hline & Vascular & Hemangioma/haemangioendothelioma/tufted angiomas \\
\hline & & Venous malformation \\
\hline & & Lymphangioma \\
\hline & & Infantile myofibromatosis \\
\hline \multirow[t]{7}{*}{ Malignant } & Primary & Ewing sarcoma/PNET* \\
\hline & & Rhabdomyosarcoma/Leiomyosarcoma/Liposarcoma \\
\hline & & Osteosarcoma/Chondrosarcoma \\
\hline & & Malignant peripheral nerve cell tumour \\
\hline & Secondary & Neuroblastoma \\
\hline & & Hepatoblastoma \\
\hline & & Leukaemia/Lymphoma \\
\hline
\end{tabular}

*PNET, primitive neuroectodermal tumour

Osteochondroma is the most common rib tumor presenting as a non-tender, hard mass [7,8]. US shows an exophytic bone lesion with a hypoechoic cartilage cap [7].

Vascular masses are usually associated with change in colour of the overlying skin. Haemangiomas are the most common vascular lesions in infancy and childhood. Typically, they are well circumscribed, but on grey-scale may show heterogeneous echogenicity with non-specific features. However, haemangiomas demonstrate high vessel density and high Doppler frequency shifts can be diagnosed on US with high specificity and sensitivity when both criteria are met [3,5-7].

Other vascular malformations, such as haemangioendothelioma, tufted angiomas and infantile myofibromatosis may have similar US appearances, without meeting both criteria [3]. Thus, additional imaging studies are necessary, including CT or MRI.

Venous malformations are characterized by spongelike serpiginous channels and anechoic cystic spaces [3, $5-7]$. In contrast to haemangiomas, the venous flow is low and often not detectable on Doppler US [6,7]. In venous malformations, phleboliths may be identified on US [6,7].

Lymphatic malformations are seen on US as multiple, cystic lesions with internal septae, without internal flow $[3,6,7]$. Although typically anechoic, their echogenicity will increase after infection or haemorrhage [6,7]. Lymphangioma is more commonly found in the axilla [6].
Although US offers a rapid, first-line assessment of benign solid masses of the chest wall, MRI is often the best method for their evaluation if malignancy is suspected [8].

\section{Malignant chest wall tumours}

Malignant chest wall tumours are uncommon in children (fig 4, fig 5) and metastatic tumours are more common than primary neoplastic lesions (Table I) [8].

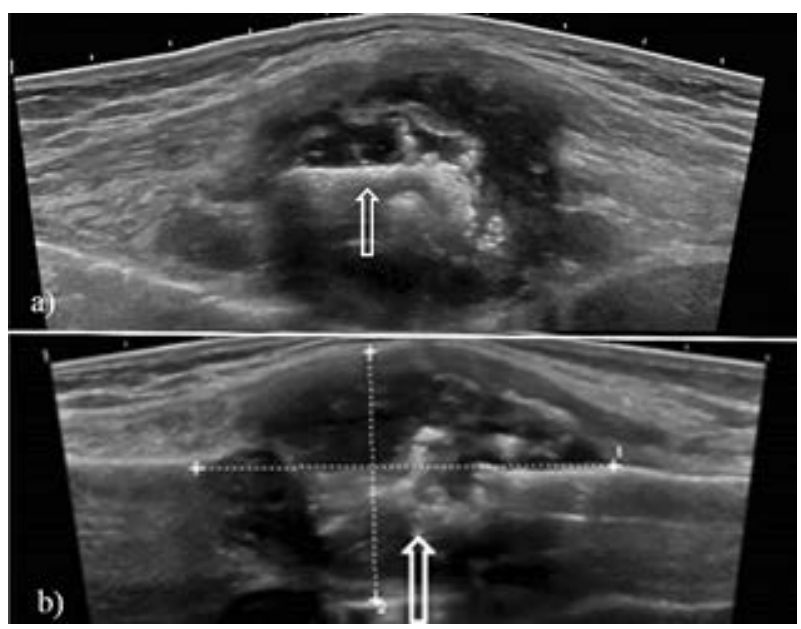

Fig 4. Hodgkin lymphoma in a 13 y/o girl. Retrosternal inhomogeneous hypoechoic tumour with fracture and destruction of the sternum (arrow), transversal (a) and longitudinal (b) plane. 


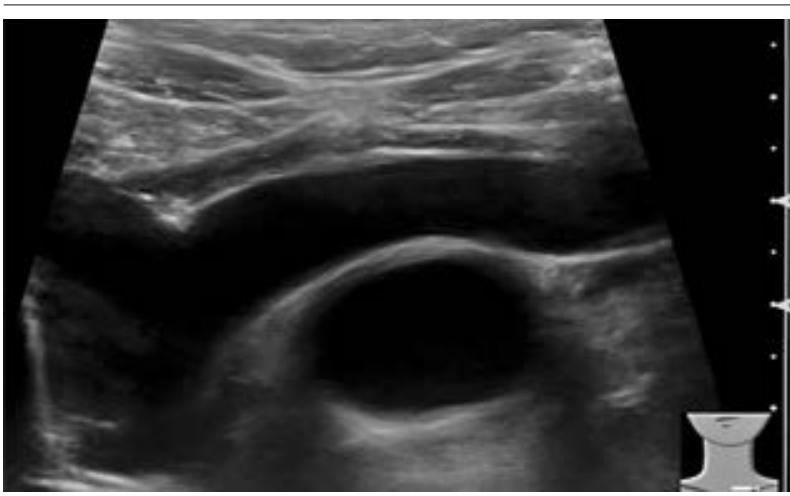

Fig 5. No thymus after heart surgery, 10-year-old boy with Down syndrome.

Clinically, malignant chest wall tumours are associated with pain and tenderness [3]. On US, they demonstrate heterogeneous echogenicity with increased Doppler flow [6]. The cortex of the adjacent bony structures may show abnormal thickening or disruption $[3,6]$. US may facilitate histological diagnosis through US-guided biopsy.

\section{MEDIASTINUM}

\section{Examination technique}

The mediastinum is imaged in the parasternal longitudinal section and scanned by tilting the ultrasound transducer. The same applies to the parasternal cross-sections in the intercostal space. Since the sternum of premature infants and young newborns is mostly cartilaginous, it can be penetrated by US when scanning in a trans-sternal approach. For older children, jugular or infra-clavicular approach is used. More superficial structures, such as the thymus, can be examined best by using a high-frequency linear transducer. Structures of the mediastinum require deeper penetration in older children with a low-frequency sector transducer. The mediastinum is the intrathoracic and extrapleural space between the lungs, containing organs from different systems, such as the thymus, trachea, oesophagus and lymph nodes. The heart and major thoracic vessels are also a part of it, but will not be considered in this publication.

\section{Thymus}

The thymus is horseshoe-shaped on cross-sectional images, with the aorta and the pulmonary artery found dorsally on the right and left side respectively. In longitudinal section images, it appears triangular to oval and is found cephalic to the aortic arch and the heart. The thymus is homogeneous in echogenicity and surrounded by a fibrous capsule, which can be visualised as an echogenic boundary layer. When examined with a high-resolution transducer, it shows echogenic lines and dots within the tissue, corresponding to normal septa and vessels. This typical echo texture allows thymic tissue to be differentiated from pathological changes in the mediastinum. The echogenicity resembles to that of the liver, sometimes being slightly more echogenic. When compared to the thyroid though, thymus is less echogenic. In older children, the thymus becomes gradually more echogenic. The thymus tissue is very soft and as result the organ demonstrates intermittent deformation, caused by the contraction of the heart. This can be assessed in real-time with US.

\section{Thymic aplasia / hypoplasia}

Thymus aplasia is characterised by absence of thymus tissue (fig 6). This occurs with immune deficiency syndrome, especially Di-George syndrome. In this case, US is the method of first choice to demonstrate the characteristic absence of thymus.

Thymic hypoplasia is very difficult to definitively diagnose due to insufficient standard values. It is only justified to raise the possibility of thymic hypoplasia if the thymus is significantly reduced and can hardly be distinguished from adjacent structures (fig 7).

\section{Thymic hyperplasia}

This is not an actual clinical entity, hence the name "physiological thymic hyperplasia". The question often arises when a chest X-ray demonstrates a shading in the area of the mediastinum, being either the thymus or another abnormal process. This question can be answered clearly by US due to the typical echo texture and echogenicity. The synchronous deformability, induced by cardiac motion is never present with atelectasis, infiltrates or tumours.

\section{Aberrant thymus}

In rare cases, thymus tissue can also be found ectopically [10-12]. Such an ectopic thymus is found pulling

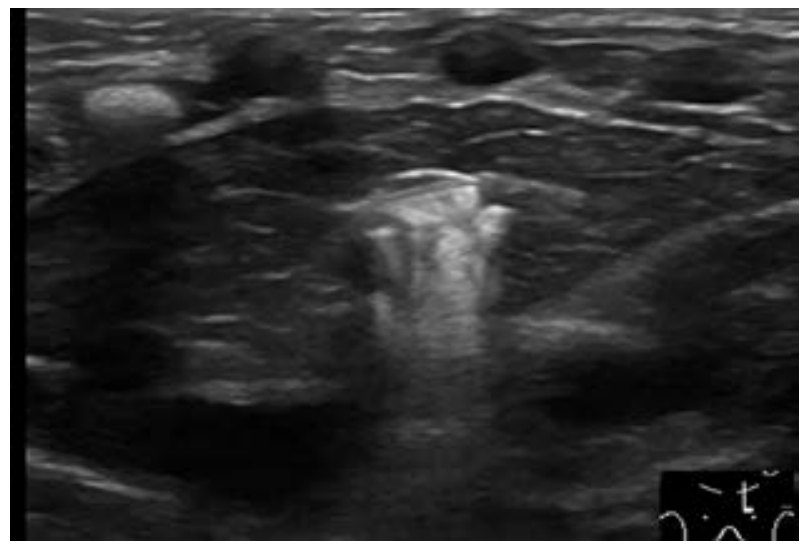

Fig 6. A 7 days-old newborn after heart catheter: thymus with air inclusions; spontaneous recovery after a few days. 


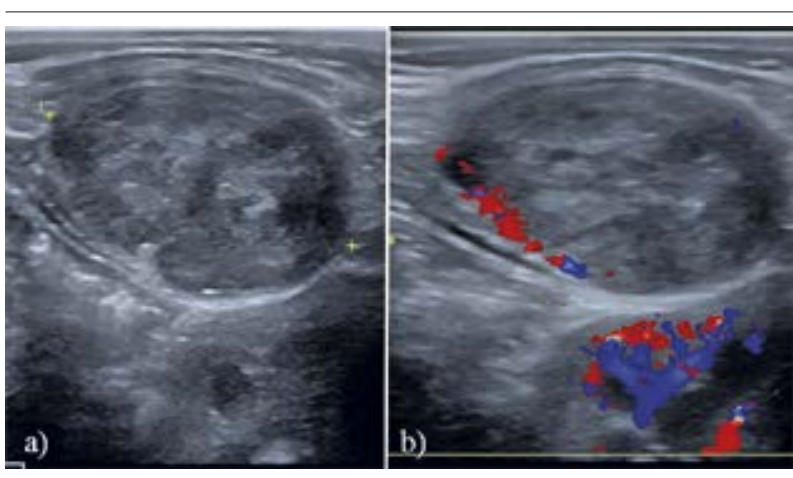

Fig 7. Fibrosarcoma, 10 months old boy, left dorsolateral chest wall tumor adjacent to the lower rib edge. B-Mode: inhomogeneous almost smoothly limited (a). Colour Doppler: only low blood flow peripheral (b).

along the thymopharyngeal duct from the lower jaw to the mediastinum. Such a thymus usually remains asymptomatic, but it can lead to vascular compression and difficulty swallowing when displaced.

\section{Structural changes of the thymus}

Structural changes in childhood are very rare. These include the thymoma (paraneoplastic syndrome), thymus carcinoma and lymphosarcoma. Leukaemia can also lead to structural changes in the thymus. These diseases cannot be differentiated with US. The thymus is usually shown relatively larger in size. The echogenicity is mostly heterogeneous, possibly due to cystic parts. The echogenicity can be increased but also reduced.

\subsection{Lymphomas}

Lymphomas are usually located in the anterior and middle mediastinum, hence often causing shift of the large vessels.

The position and width of the trachea is important. Especially in T-cell lymphoma rapid growth is possible and decision for early therapy is necessary before the trachea is collapsed. Imaging findings include both enlarged lymph nodes and diffuse thymus infiltration. Contrary to inflammatory changes, lymphomas are less vascularised on colour Doppler technique, but a confident differentiation is not possible with Doppler technique only. On US, lymph nodes in lymphomas appear mostly multiple, rounded oval and hypoechoic. They are often polycyclic limited and are in the vicinity of the large vessels. Differentiation from other solid processes is not always possible. US can be used to observe thoracic wall or pericardial infiltration and to look for infradiaphragmal lymph nodes.

\subsection{Solid masses}

Solid masses are rare in childhood, and differentiation based on US alone is not possible. They are usually hyperechoic and heterogeneous, often containing very echogenic parts with acoustic shadowing, representing calcifications. There may also be some cystic compo- nents. A better classification can be achieved through the precise localisation of the lesion. Teratomas $(15 \%$ of the mediastinal masses), teratocarcinomas, chorionic carcinomas, yolk sac tumours and thymolipomas (mostly relatively homogeneous) are located in the anterior and middle mediastinum.

Neuroblastoma, ganglioneuroma and neurofibroma are usually found in the posterior mediastinum next to the vertebral column and can be visualised from a posterior view (sitting position).

\subsection{Cystic masses}

Such lesions are shown sonographically if they are liquid-filled or empty, with fluid appearing hypoechoic on US. Differentiation is only feasible based on the topographical location.

The most common cystic masses are thymic cysts and mediastinal lymphangiomas. They usually have smooth walls. Bleeding can occasionally make the contents of the cyst appear more echogenic. Ultrasound may be helpfully to differentiate bronchogenic cyst from layered foregut duplication cysts (oesophageal duplication) [13].

\section{Trachea}

The echopoor tracheal cartilage and the ventral wall can be visualised sonographically. It is possible to measure the thickness of the cartilage, the width of the trachea $[14,15]$ or to evaluate movements of the trachea. US may be helpfully to differentiate bronchogenic cyst from layered foregut duplication cysts (oesophageal duplication). Haemangioma can be visualised in the subglottic position. Beside the vertebral column, neurogenic cysts are possible. The adjacent air filling of the trachea leads to dorsal sound cancellation and dirty shadowing. Thus, pathological changes in the trachea are difficult to evaluate.

The position of endotracheal tubes can be checked using US. With a subglottic visualisation of the trachea, the width of the sound cancellation correlates very well with the size of the tube to be selected [16]. The tube is shown in cross-section as two short, parallel echogenic lines. In longitudinal section, the tube below the ventral wall of the trachea is shown as longitudinal, parallel echogenic lines. The movement of the tube facilitates identification. This means that both the depth of the intubation and the faulty intubation can be displayed.

\section{Diaphragm}

US of the diaphragm is a reliable method for evaluation of anatomy and function of the diaphragm. It was firstly described in 1969 by Cohen followed by Haber in 1975 [17], who showed that US is superior to fluor- 
oscopy in assessing diaphragmatic movement disorders [18]. The diaphragm has a crucial role in breathing. Multiple methods for evaluation were developed including fluoroscopic sniff test, electromyography and nerve conduction, which are used in detection of diaphragmatic dysfunction. However, these are time-consuming and procedure-related complications might occur [19]. US evaluation of the diaphragm has proved to be useful in patients with suspicion of diaphragm paralysis for decades. From diaphragm paralysis [20], to the evaluation of respiratory function [21], diaphragm US has been confirmed to be a valuable technique, useful in the diagnosis of different signs and symptoms like paradoxical respiration, dyspnoea or asymmetric radiographic findings. Other conditions that can be diagnosed by US include diaphragmatic hernias, eventrations, peridiaphragmatic abscess, thoracic tumours, pleural collections and lesions causing diaphragm paralysis [17].

\section{Examination technique}

Diaphragmatic US is performed ideally with the patient in the supine position or in a sitting position if the former is not possible, e.g., in children with a neuromuscular disorder.

Lateral parts of the diaphragm can be imaged in an axillary longitudinal section and appear similar to muscles in being generally hypoechoic. Above the spleen and the liver, the diaphragm appears as a thin hypoechoic three-layered muscular structure when using high frequency transducers. The interface between air-filled tissue (lung) and solid organs (liver, spleen) is generated by changes in the acoustic impedance and appears as a bright echo line using low frequency transducers. This so-called bright diaphragm line does not represent the diaphragm itself but an interface. The position of the diaphragm and respiratory diaphragmatic movement are the focus of the evaluation. The best examination position is the sub-diaphragmatic longitudinal view along the anterior axillary line. Comparison between the left and right side can be performed by tilting the transducer to the cranial sub sternal cross-section. In this case, the right side of the diaphragm can be very well seen while showing the left side can be challenging due to the air-filled stomach. Because of the necessary depth of penetration, low-frequency convex transducers (3-9 MHz) should be used. By using M-mode, the mobility of the diaphragm can be shown and measured.

Deep breathing might be difficult in non-compliant children [17]. The linear, high frequency transducers (7$17 \mathrm{MHz}$ ) are primarily used for the diaphragmatic muscle assessment and the convex probe (micro convex for children and thin patients) or a sectorial for smaller children [22]:
- The anterior central view from the subxiphoid window is recommended for the left and right hemidiaphragm. This view allows the comparison between the two hemidiaphragms and their movements, using the sectorial or a micro convex probe, in B mode, transversely positioned transabdominal, cranially upright oriented [19].

- The lateral views, in the so-called apposition area, between the axillar anterior line and mid-clavicular line, with the linear high frequency transducer, positioned longitudinally, perpendicular to the lower ribs;

- The right hemidiaphragm is easily seen through the liver window, while the left diaphragm is not easy through the spleen window, because of stomach [23] or other abdominal content [20].

A good image may be obtained between the midclavicular and anterior axillary lines in the subcostal area and by directing the probe medially and cranially to achieve the best view of the right hemidiaphragm at the apposition zone [24]. For the posterior view, in the sitting position, using a convex probe, the renal parenchyma may conduct the US to enable the diaphragm to be assessed in children, but the window might be smaller and not possible in every child as it is thoracic wall size dependent [17].

The intercostal approach can be used but the intercostal space is different in children, compared to adults. However, the preferred intercostal spaces in adults between $7^{\text {th }}-8^{\text {th }}$ rib or $8-9^{\text {th }}$ rib even $9-10^{\text {th }}$ are potentially applicable in supine children [17]. The estimation of the lateral diaphragm parts is important, because of their main role in breathing compared to the anterior central zone which has less effect on inspiration [25]. Diaphragmatic US has a few limitations: firstly, standard reference parameters such as thickness are only available in some populations; secondly, it is difficulty to visualize the left diaphragm in overweight children. Lastly, assessment of the diaphragm motion can be affected by inspiratory effort and interposition of abdominal organs [17].

\section{Diaphragm parameters}

The measurable diaphragm parameters are: diaphragmatic thickness excursion and thickening fraction. The first is a static parameter while the others are dynamic parameters.

The diaphragm thickness (DT) is increased in inspiration and decreases in expiration. DT is well correlated with body weight in children and adults. Other parameters like trans diaphragmatic pressure is higher in children compared to adults [26]. Normal DT value scales are available in healthy adults [27] and children [26] but only in limited populations. Atrophy of the diaphragm 
occurs in ventilated children with acute respiratory failure, especially in those receiving neuromuscular blockers [28]. DT is a very important parameter for detection of diaphragmatic atrophy of mechanically ventilated children [29] and is a reliable prognostic indicator for ventilator-induced diaphragmatic atrophy [29] and for the risk for extubation failure in children [30]. US can be used for diagnosing atrophy secondary to other diseases such as myodystrophy, malnutrition, trauma, compression and associated breathing disorders with restrictive dysfunction, malnutrition and electrolyte disturbances (hypophosphatemia, hypokalaemia, hypocalcaemia) [31].

Diaphragmatic excursion (DE) is assessed by Mmode US, measuring the distance of diaphragm movement between end inspiration and end expiration [32] and normal reference values exist in small adult populations [33] and also a few in children [20]. Decreased DE can be suggestive of a number of diaphragm disorders, such as neuromuscular diseases (including hypocalcaemia, hypokalaemia, hypophosphatemia [31]), neuropraxia secondary to induced hypothermia, ventilated patients and pathology in the vicinity, from lung and pleura (pneumonia, pleural effusions) or abdomen (peritonitis).

Diaphragm thickening fraction (DTF) is defined as the fraction of the difference between the thicknessat-end inspiration and thickness-at-end expiration / thickness-at-end-expiration [19]. The increased DTF is strongly correlated with spontaneous breathing fraction and can predict weaning success, correlated with an increased percentage of successful extubation [34], even in post transplanted patients [35].

\section{Diaphragm motility}

The evaluation of diaphragm motility is essential when there is a suspicion of diaphragmatic paralysis. This could be performed by chest X-ray, which can demonstrate elevation of a hemidiaphragm, but does not provide information on diaphragm motion. CT would be more specific in detecting the structural changes but provide less information on motility and expose the patients to increased ionizing radiations. Diaphragm motility might be evaluated by MRI which does not involve ionizing radiation, but it is more time consuming and expensive and sedation might be required [19,36,37].

It has been shown that US is a "highly sensitive method of demonstrating generalised or localised abnormalities of diaphragmatic motion" [18] and new data suggest that diaphragm motility is a predictor for effective extubation in children [38], confirming the importance of US for diaphragm evaluation. One of the most important indications for diaphragm US is after cardiac surgery where there is a suspicion of diaphragm paralysis. The diaphragm paralysis can be promptly evaluated by
US and it has been shown to be superior to fluoroscopy [39].

\section{Specific pathological findings}

Diaphragmatic conditions range from congenital malformations such as diaphragmatic hernia to diaphragm paralysis secondary to phrenic nerve injury in cardiac surgery or muscular dystrophies and neuromuscular disorders [40]. Detecting traumatic diaphragm rupture using US has been suggested as an additional examination in "Focused Abdominal Sonography for Trauma" (FAST) examination for adults and children [41,42].

In diaphragmatic impairment, lung function assessed by spirometry reveals a restrictive pattern, but spirometry is challenging to perform in children, particularly in those with neuromuscular diseases. It has been found that a significant correlation exists between lung volumes and diaphragm function in adults [43] and a linear correlation between diaphragmatic thickening fraction and lung volumes was demonstrated in children [27]. However, this correlation was not confirmed in a study evaluating the relationship between body plethysmography and diaphragm respiratory course in adults [44].

Congenital diaphragmatic disorders include anatomical defects such as Bochdalek and Morgagni congenital hernia, eventration, diaphragmatic agenesis and functional disorders including diaphragm paralysis, atrophy, posttraumatic and iatrogenic injury during surgery and neuromuscular diseases, all have important effects in childhood [27].

Congenital diaphragmatic hernia $(\mathrm{CDH})$ is one of the most frequent major congenital anomalies, with a significant postnatal mortality rate due to acute complications or development of pulmonary hypertension [45]. Diaphragmatic hernia can be easily diagnosed with US. Chest X-ray is the first imaging modality postnatally. In fact, most cases are diagnosed prenatally by foetal US combined with foetal MRI for risk evaluation (e.g., lung volumetry). $\mathrm{CDH}$ can be diagnosed on chest $\mathrm{X}$-ray when gas containing bowel or stomach are projected over the lung fields; if the solid organs such as liver are involved. US is the method of choice for diagnosis and can differentiate $\mathrm{CDH}$ from pleural effusion in post-operative patients [22]. US before corrective surgery can accurately evaluate the size of the defect in $\mathrm{CDH}$ and can provide anatomical information, which can consequently influence the surgical approach [46].

\section{US GUIDED/ASSISTED INTERVENTIONS}

As discussed previously, US has an important role in assessing all thoracic compartments: chest wall, pleura, lung, mediastinum and diaphragm. Thus, interventional 
procedures involving any of these compartments are often best and most safely performed using US guidance or assistance. US guided drainage of chest wall or lung abscesses aids the microbiological diagnosis, while offering a minimally invasive therapeutic opportunity [3]. Accessible mediastinum lymph nodes and solid tumours of the chest wall or lung may be biopsied via US guidance $[3,4,6,7]$. US guidance is preferred for peripheral lung biopsy for both children and adults [38,47-49]. The utility and low radiation burden of US guided insertion of arterial and venous catheters is well established [5,50,51]. In addition, venous malformations of the chest wall may benefit from US guided sclerotherapy $[6,52]$. Diagnostic and therapeutic US guided drainage of pleural effusion and empyema is well described in the literature $[53,54]$. As per recent guidelines, fibrinolytic treatment is initiated via transthoracic catheter [55]. The use of US guided insertion of small-bore pig-tail catheters appears safe and efficient both for symptomatic pleural effusion [56] and empyema [57]. Although, US is not usually employed for identifying pneumothorax in some cases it may be a more accessible and rapid method of diagnosis and treatment [58].

US guided minimally invasive therapeutic approaches are employed for congenital pulmonary airway malformation (CPAM) with sequestration, both in the foetal and neonatal period $[6,7,59]$. In a case series, the authors describe the occlusion of the feeding vessel in utero via interstitial laser, thrombogenic coil embolization and radio-frequency ablation [59].

\section{Interventions in the pleural space}

US is the most specific and sensitive imaging study used to confirm the presence and type of pleural effusion, as well as the optimal site for tube placement [55]. It can also signal complications such as the presence of septation or debris within the pleural space [55]. The size of the pleural effusion, cultures and respiratory compromise guide the decision for drainage of the pleural effusion [60] (Table II).

In children, $10 \mathrm{~F}$ and $12 \mathrm{~F}$ drainage tubes are considered equally effective. When choosing the site of the thoracocentesis, whether diagnostic or therapeutic, one should always have in mind the "safe triangle". Samples of the pleural effusion must be sent for biochemical and microbiologic testing. If there is suspicion that the pleural effusion is not secondary to infection, cytological analysis is mandatory.

When therapeutic thoracocentesis is performed, the drainage tube is placed to one-way suction, ranging from -5 to $-20 \mathrm{~cm}$ of water [55].

Currently, there is no consensus regarding pleural drainage volumes in children. Volumes equal to $10 \mathrm{ml} /$ $\mathrm{kg}$ with a maximum of 1.51 are generally considered safe. Re-expansion pulmonary oedema (RPE) is the most feared complication of large and rapid pleural effusion drainage. Still, RPE is rare in children.

Intrapleural fibrinolytic agents are used successfully for pleural drainage. There are several protocols used for pleural sclerotherapy using either urokinase or tissue plasminogen activator $[61,62]$.

Pleuritic pain must be managed with analgesics in order to ensure appropriate expansion of the lung after the procedure. When the output of the chest tube decreases to $<1 \mathrm{ml} / \mathrm{kg} / 24$ hours, it may be removed [61].

\section{ARTEFACTS}

Recognition of artefacts is crucial in order to avoid misinterpretation of the findings and false diagnosis. Physicians should thus familiarise themselves with the terminology and nature of these technical phenomena.

The term "beam width artefact" describes the visualisation of echoes generated from a point lying outside the US beam, inside the imaging plane and represents an expression of US lateral resolution. To understand this artefact, it is important to remember that the US beam is bow-tie shaped, being narrowed at the level of the focus point and then widens reaching a width larger than the probe width. As a result, a reflection originating from a point lying outside the width of the transducer but within the widened width of the beam will be erroneously displayed inside the field-of-view. In practice, this explains why bright echoes can be falsely displayed within anechoic structures such as the gallbladder or an anechoic pleural effusion. The solution to this problem is always to keep the focal point at the level of structure under examination, using multiple focal zones or placing the point of interest at the centre of transducer. In that way, the beam

Table II. Pleural drainage decision.

\begin{tabular}{lll}
\hline Size of effusion (lateral decubitus) & Culture & Pleural drainage $+/$ - fibrinolysis \\
\hline Small: $<10 \mathrm{~mm}$ or opacifies $<25 \%$ of hemithorax & Unknown/Negative & No \\
Moderate: $>10 \mathrm{~mm}$ or opacifies $<50 \%$ of hemithorax & Negative/Positive & No (no respiratory compromise and no empyema) \\
& & Yes (respiratory compromise or empyema) \\
Large: Opacifies $>50 \%$ of hemithorax & Positive (empyema) & Yes \\
\hline
\end{tabular}


will have its narrowest width at the level of examination, visualising only the area of interest and leaving adjacent echoes outside the imaging field $[63,64]$.

The false visualization of echoes external to the imaging field can also be caused by the "side lobe artefact". The radial expansion of piezoelectric crystals gives rise to multiple low-amplitude waves of energy situated laterally to the main array. When these waves encounter strong reflectors, the returning signals are shown as originating from within the main imaging field. This artefact can also result in the false visualization of echoes within anechoic structures such as the gallbladder or a pleural effusion [63]. Potential solutions include decreasing gain, using harmonics or different acoustic windows [64]. The reflection of the US beam produces a number of artefacts. When the US beam is completely reflected by a strongly reflective surface such as bone or calculi, the absence of any echo signal behind these surfaces is known as 'acoustic shadowing". In the lungs, 'acoustic shadowing" occurs with the ribs hindering evaluation of underlying pathology.

When the US beam encounters two parallel highly reflective structures, part of the beam is repeatedly reflected between the two structures. Some of those will reach the transducer and will appears abnormally brightly as a result of the time the beam has been reflected. This is called the "reverberation artefact" and can be seen in lung US when the beam hits catheters visualised as two parallel echogenic lines.

The "comet tail" artefact is similar to the above, being only different in that it appears triangular with echoes gradually narrowing due to the decreasing attenuation of each reflected echo. The difference results from the fact that the strong reflective areas are closely situated and thus hardly discernible by the beam.

The "ring down" artefact is another form of artefact produced by the sound energy generated by fluid trapped between air-bubbles. This appears as a white vertical straight line below the gas bubble and can be seen in lung abscesses or colonic loops under the diaphragm. Similar to the mechanism of ring-down artefact, aggregates of gas may give rise to "dirty shadowing", consisting of an area without useful signal. This can be seen with normal lung parenchyma and gas bubbles in lung abscesses $[63,64]$ Reverberation and comet tail artefact can be mitigated by reducing gain or using a different acoustic window. On the contrary, if these artefacts need to be accentuated in order to better detect pathologic gas, the spatial compounding function should be turned off [64].

The "mirror image" artefact is seen when the echo generated from a strong reflective surface such as the diaphragm encounters another object during its path back to the transducer. Part of the echo will be reflected back a second time to create a false secondary echo which creates a 'mirror image' of the true lesion on the opposite side of the diaphragm. The commonest example is seen as reflection of a liver lesion into the thorax. This artefact may also result in the reflection of abdominal ascites into the hemi thorax, thus mimicking a pleural effusion [63,64].

Another important artefact is the "speed displacement" artefact, in which US travels with different speed through different materials. As a result, the time required for a reflected signal to reach the transducer is different. As the ultrasound machine assumes an equal speed for all echoes reflected, this causes the artefactual visualization of echoes in deeper locations. In lung US, this explains why the diaphragm may appear discontinuous with some of its segments being visualised deeper because of different speed of sound through fatty parts of the liver [63].

In contrast to acoustic shadowing, acoustic enhancement occurs when the US beam travels through an anechoic structure such a pleural effusion, beam attenuation is lower than that of the adjacent beam, meaning that the next reflected echo will be visualised brighter [63]. An important artefact arising from the lung-pleura interface is call B-line artefact (BLA). In healthy children with a normally aerated lung, nothing is visible on US other than horizontal hyperechoic lines below the pleural lines known as $A$ lines, these are reverberation artefacts. In patients with alveolar - interstitial syndrome, a disease involving the alveolar space and pulmonary interstitial, this instead appears as multiple narrow hyperechoic laser-beam which reaches up to the edges of the screen. This is known as a "comet tail" and is also known as BLA [65], however, the latter is the preferred term when referring to the lung. Both, mild or severe alveolar - interstitial syndrome can lead to this artefact. In patients with localised disease, the involved lobe can also be localised based on correlation of the intercostal rib space and presence of the "comet tail" artefact with sensitivity ranging from $86 \%$ to $93 \%$ and specificity of $87 \%$ to $96 \%$ [66]. A number of factors have been described to influence the detection of BLA $[67,68]$. Although BLA was initially described in alveolar - interstitial syndrome, any conditions which cause loss of lung aeration peripherally leading to increased density will give rise to BLA.

Artifacts in contrast-enhanced ultrasound (CEUS)

Contrast-enhanced ultrasound (CEUS) is a well-established technique for many abdominal organs in adults but has also been shown to have lung applications [69]. In Europe, intravenous CEUS in children is still performed off-license. Most CEUS artefacts occur in abdominal applications but they can also affect lung imaging. 
Non-linear artefacts or incomplete suppression may be encountered with highly echogenic interfaces or structures, which are projected on the contrast-specific image. These signals should not be perceived as true enhancement and can be differentiated from microbubbles signal by comparing the contrast-specific and low-mechanical index B-mode image prior to contrast administration, as they will appear identical in both images. In the case of lung US, gas bubbles from abscesses can be visualised as echogenic areas projected on contrast-specific images. When assessing a lung consolidation, it should be kept in mind that near-field signal loss can be caused by the disruption of microbubbles incurred by the higher acoustic pressure in this part of the imaging plane. Imaging plane signal loss can be seen when scanning on the same plane for a long period of time. The continuous acoustic pressure disrupts microbubbles. This mainly interferes with liver examinations for the characterization of focal lesions and is less common in the lung where the transducer typically sweeps through areas of consolidation or pleural pathology to detect necrosis. High doses of microbubbles cause shadowing similar to ribs, due to the attenuation of beam. This can be observed in intracavitary applications of CEUS, where the solution to be administered needs to be carefully reconstituted, including only a drop $(0.1 \mathrm{ml})$ of microbubbles [70,71].

\section{Conclusion}

The series of reviews is intended to summarize and illustrate current knowledge on thoracic ultrasound in paediatric patients. The reader is warmly welcome to stimulate a discussion and to illustrate the initiated WFUMB Atlas.

\section{Conflict of interest: none}

\section{References}

1. Jaworska J, Buda N, Ciuca IM, et al. Ultrasound of the pleura in children, WFUMB review paper. Med Ultrason 2021. doi: $10.11152 / \mathrm{mu}-3058$.

2. Dietrich CF, Buda N, Ciuca IM, et al. Lung ultrasound in children, WFUMB review paper (part 2). Med Ultrason 2021. doi: 10.11152/mu-3059.

3. Mong A, Epelman M, Darge K. Ultrasound of the pediatric chest. Pediatr Radiology 2012;42:1287-1297.

4. Joshi P, Vasishta A, Gupta M. Ultrasound of the pediatric chest. Br J Radiol 2019;92:20190058.

5. Cox M, Soudack M, Podberesky DJ, Epelman M. Pediatric chest ultrasound: a practical approach. Pediatr Radiol 2017;47:1058-1068.

6. Coley BD. Chest sonography in children: current indications, techniques, and imaging findings. Radiol Clin North Am 2011;49:825-846.
7. Supakul N, Karmazyn B. Ultrasound of the pediatric chestthe ins and outs.Semin Ultrasound CT MR 2013;34:274285.8 .

8. Newman B. Thoracic neoplasms in children. Radiol Clin North Am 2011;49:633-664.

9. Milos RI, Moritz T, Bernathova M, et al. Superficial desmoid tumors: MRI and ultrasound imaging characteristics. Eur J Radiol 2015;84:2194-2201.

10. Yildiz AE, Elhan AH, Fitoz S. Prevalence and sonographic features of ectopic thyroidal thymus in children: A retrospective analysis. J Clin Ultrasound 2018;46:375-379.

11. Yildiz AE, Ceyhan K, Siklar Z, et al. Intrathyroidal Ectopic Thymus in Children: Retrospective Analysis of Grayscale and Doppler Sonographic Features. J Ultrasound Med 2015;34:1651-1656.

12. Bang MH, Shin J, Lee KS, Kang MJ. Intrathyroidal ectopic thymus in children: A benign lesion. Medicine (Baltimore) 2018;97:e0282.

13. Balakrishnan K, Fonacier F, Sood S, Bamji N, Bostwick H, Stringel G. Foregut Duplication Cysts in Children. JSLS 2017;21:e2017.00017.

14. Wanner MR, Marine MB, Dahl JP. Ultrasound diagnosis of tracheal cartilaginous sleeve in a patient with Pfeiffer syndrome. Pediatr Radiol 2018;48:1814-1816.

15. Diwakar A, Adam RJ, Michalski AS, et al. Sonographic evidence of abnormal tracheal cartilage ring structure in cystic fibrosis. Laryngoscope 2015;125:2398-2404.

16. Gollu G, Onat Bermede A, Khanmammadov F, et al. Use of ultrasonography as a noninvasive decisive tool to determine the accurate endotracheal tube size in anesthetized children. Arch Argent Pediatr 2018;116:172-178.

17. Sarwal A, Walker FO, Cartwright MS. Neuromuscular ultrasound for evaluation of the diaphragm. Muscle Nerve 2013;47:319-329.

18. Haber K, Asher M, Freimanis AK. Echographic evaluation of diaphragmatic motion in intra-abdominal diseases. Radiology 1975;114:141-144.

19. Fayssoil A, Behin A, Ogna A, et al. Diaphragm: Pathophysiology and Ultrasound Imaging in Neuromuscular Disorders. J Neuromuscul Dis 2018;5:1-10.

20. Riccabona M, Sorantin E, Ring E. Application of M-mode sonography to functional evaluation in pediatric patients. Eur Radiol 1998;8:1457-1461.

21. Riccabona M. Ultrasound of the chest in children (mediastinum excluded). Eur Radiol 2008;18:390-399.

22. Trinavarat P, Riccabona M. Potential of ultrasound in the pediatric chest. Eur J Radiol 2014;83:1507-1518.

23. Cohen E, Mier A, Heywood P, Murphy K, Boultbee J, Guz A. Excursion-volume relation of the right hemidiaphragm measured by ultrasonography and respiratory airflow measurements. Thorax 1994;49:885-889.

24. El-Halaby H, Abdel-Hady H, Alsawah G, Abdelrahman A, El-Tahan H. Sonographic Evaluation of Diaphragmatic Excursion and Thickness in Healthy Infants and Children. J Ultrasound Med 2016;35:167-175.

25. Houston JG, Morris AD, Howie CA, Reid JL, McMillan N. Technical report: quantitative assessment of diaphragmatic 
movement--a reproducible method using ultrasound. Clin Radiol 1992;46:405-407.

26. Rehan VK, McCool FD. Diaphragm dimensions of the healthy term infant. Acta Paediatr 2003;92:1062-1067.

27. McCool FD, Tzelepis GE. Dysfunction of the diaphragm. N Engl J Med 2012;366:932-942.

28. Goligher EC, Laghi F, Detsky ME, et al. Measuring diaphragm thickness with ultrasound in mechanically ventilated patients: feasibility, reproducibility and validity. Intensive Care Med 2015;41:642-649.

29. Johnson RW, Ng KWP, Dietz AR, et al. Muscle atrophy in mechanically-ventilated critically ill children. PLoS One 2018;13:e0207720.

30. Terhart MN, Hanekom S, Lupton-Smith A, Morrow B. Reliability of ultrasonic diaphragm thickness measurement in mechanically ventilated infants and children: A pilot study. South Afr J Crit Care (Online) 2018;34:22-27.

31. McParland C, Resch EF, Krishnan B, Wang Y, Cujec B, Gallagher CG. Inspiratory muscle weakness in chronic heart failure: role of nutrition and electrolyte status and systemic myopathy. Am J Respir Crit Care Med 1995;151:11011107.

32. Turton P, ALAidarous S, Welters I. A narrative review of diaphragm ultrasound to predict weaning from mechanical ventilation: where are we and where are we heading? Ultrasound J 2019;11:2.

33. Boussuges A, Gole Y, Blanc P. Diaphragmatic motion studied by m-mode ultrasonography: methods, reproducibility, and normal values. Chest 2009;135:391-400.

34. Glau CL, Conlon TW, Himebauch AS, et al. Progressive Diaphragm Atrophy in Pediatric Acute Respiratory Failure. Pediatr Crit Care Med 2018;19:406-411.

35. Sharma A, Karna ST, Tandon M, et al. Use of ultrasoundguided preoperative diaphragmatic thickness as a predictor of postoperative weaning failure in recipients and donors scheduled for living donor liver transplant surgery. Saudi J Anaesth 2018;12:406-411.

36. Caraiani C, Dong Y, Rudd AG, Dietrich CF. Reasons for inadequate or incomplete imaging techniques. Med Ultrason 2018;20:498-507.

37. Caraiani C, Petresc B, Dong Y, Dietrich CF. Contraindications and adverse effects in abdominal imaging. Med Ultrason 2019;21:456-463.

38. Lee EP, Hsia SH, Hsiao HF, et al. Evaluation of diaphragmatic function in mechanically ventilated children: An ultrasound study. PLoS One 2017;12:e0183560.

39. Sanchez de Toledo J, Munoz R, Landsittel D, et al. Diagnosis of abnormal diaphragm motion after cardiothoracic surgery: ultrasound performed by a cardiac intensivist vs. fluoroscopy. Congenit Heart Dis 2010;5:565-572.

40. Dube BP, Dres M. Diaphragm Dysfunction: Diagnostic Approaches and Management Strategies. J Clin Med 2016;5:113.

41. Blaivas M, Brannam L, Hawkins M, Lyon M, Sriram K. Bedside emergency ultrasonographic diagnosis of diaphragmatic rupture in blunt abdominal trauma. Am J Emerg Med 2004;22:601-604.
42. Gangahar R, Doshi D. FAST scan in the diagnosis of acute diaphragmatic rupture. Am J Emerg Med 2010;28:387.e1-3.

43. Ayoub J, Metge L, Dauzat M, et al. Diaphragm kinetics coupled with spirometry. M-mode ultrasonographic and fluoroscopic study; preliminary results. J Radiol 1997;78:563-568.

44. Scott S, Fuld JP, Carter R, McEntegart M, MacFarlane NG. Diaphragm ultrasonography as an alternative to wholebody plethysmography in pulmonary function testing. $\mathrm{J}$ Ultrasound Med 2006;25:225-232.

45. Brownlee EM, Howatson AG, Davis CF, Sabharwal AJ. The hidden mortality of congenital diaphragmatic hernia: a 20-year review. J Pediatr Surg 2009;44:317-320.

46. Hattori K, Takamizawa S, Miyake Y, et al. Preoperative sonographic evaluation of the defect size and the diaphragm rim in congenital diaphragmatic hernia - preliminary experience. Pediatr Radiol 2018;48:1550-1555.

47. Jarmakani M, Duguay S, Rust K, Conner K, Wagner JM. Ultrasound Versus Computed Tomographic Guidance for Percutaneous Biopsy of Chest Lesions. J Ultrasound Med 2016;35:1865-1872.

48. Safai Zadeh E, Keber CU, Dietrich CF, et al. Perfusion patterns of peripheral pulmonary granulomatous lesions using contrast-enhanced ultrasound (CEUS) and their correlation with immunohistochemically detected vascularization patterns. JUltrasound Med 2021. doi: 10.1002/jum.1573049.

49. Safai Zadeh E, Beutel B, Dietrich CF, et al. Perfusion Patterns of Peripheral Pulmonary Lesions in COVID-19 Patients Using Contrast-Enhanced Ultrasound (CEUS): A Case Series. J Ultrasound Med 2021. doi:10.1002/ jum. 15624.

50. Bajaj M, Wells J, Liyanage A, Evans S, Hamill J. Radiation burden of pediatric ultrasound-guided percutaneous central venous access devices: A prospective cohort study. J Pediatr Surg 2018;53:802-807.

51. Jenssen C, Brkljacic B, Hocke M, et al. EFSUMB Guidelines on Interventional Ultrasound (INVUS), Part VI - Ultrasound-Guided Vascular Interventions. Ultraschall Med 2016;37:473-476.

52. Kumar S, Bhavana K, Kumar S, Kumar P. Ultrasoundguided polidocanol foam sclerotherapy for treating venous malformations. J Clin Ultrasound 2018;46:23-31.

53. Corcoran JP, Tazi-Mezalek R, Maldonado F, et al. State of the art thoracic ultrasound: intervention and therapeutics. Thorax 2017;72:840-849.

54. Safai Zadeh E, Gorg C, Dietrich CF, Gorlach J, Alhyari A, Trenker C. Contrast-Enhanced Ultrasound for Evaluation of Pleural Effusion: A Pictorial Essay. J Ultrasound Med 2021. doi:10.1002/jum.15705.

55. Feola GP, Hogan MJ, Baskin KM, et al. Quality Improvement Standards for the Treatment of Pediatric Empyema. J Vasc Interv Radiol 2018;29:1415-1422.

56. Miraglia R, Maruzzelli L, Piazza M, et al. Real-time ultrasound-guided placement of a pigtail catheter in supine position for draining pleural effusion in pediatric patients who have undergone liver transplantation. J Clin Ultrasound 2016;44:284-289. 
57. Lewis MR, Micic TA, Doull IJM, Evans A. Real-time ultrasound-guided pigtail catheter chest drain for complicated parapneumonic effusion and empyema in children 16-year, single-centre experience of radiologically placed drains. Pediatr Radiol 2018;48:1410-1416.

58. Migliaro F, Sodano A, Capasso L, Raimondi F. Lung ultrasound-guided emergency pneumothorax needle aspiration in a very preterm infant. BMJ Case Rep 2014;2014:bcr2014206803.

59. Baud D, Windrim R, Kachura JR, et al. Minimally invasive fetal therapy for hydropic lung masses: three different approaches and review of the literature. Ultrasound Obstet Gynecol 2013;42:440-448.

60. Bradley JS, Byington CL, Shah SS, et al. Executive summary: the management of community-acquired pneumonia in infants and children older than 3 months of age: clinical practice guidelines by the Pediatric Infectious Diseases Society and the Infectious Diseases Society of America. Clin Infect Dis 2011;53:617-630.

61. Sonnappa S, Cohen G, Owens CM, et al. Comparison of urokinase and video-assisted thoracoscopic surgery for treatment of childhood empyema. Am J Respir Crit Care Med 2006;174:221-227.

62. Hawkins JA, Scaife ES, Hillman ND, Feola GP. Current treatment of pediatric empyema. Semin Thorac Cardiovasc Surg 2004;16:196-200.
63. Feldman MK, Katyal S, Blackwood MS. US artifacts. Radiographics 2009;29:1179-1189.

64. Baad M, Lu ZF, Reiser I, Paushter D. Clinical Significance of US Artifacts. Radiographics 2017;37:1408-1423.

65. Lichtenstein D, Meziere G, Biderman P, Gepner A, Barre O. The comet-tail artifact. An ultrasound sign of alveolar-interstitial syndrome. Am J Respir Crit Care Med 1997; 156:1640-1646.

66. Stefanidis K, Dimopoulos S, Kolofousi C, et al. Sonographic lobe localization of alveolar-interstitial syndrome in the critically ill. Crit Care Res Pract 2012;2012:179719.

67. Dietrich CF, Mathis G, Blaivas M, et al. Lung artefacts and their use. Med Ultrason 2016;18:488-499.

68. Dietrich CF, Mathis G, Blaivas M, et al. Lung B-line artefacts and their use. J Thorac Dis 2016;8:1356-1365.

69. Sidhu PS, Cantisani V, Dietrich CF, et al. The EFSUMB Guidelines and Recommendations for the Clinical Practice of Contrast-Enhanced Ultrasound (CEUS) in Non-Hepatic Applications: Update 2017 (Short Version). Ultraschall Med 2018;39:154-180.

70. Fetzer DT, Rafailidis V, Peterson C, Grant EG, Sidhu P, Barr RG. Artifacts in contrast-enhanced ultrasound: a pictorial essay. Abdom Radiol (NY) 2018;43:977-997.

71. Dietrich CF, Ignee A, Hocke M, Schreiber-Dietrich D, Greis C. Pitfalls and artefacts using contrast enhanced ultrasound. Z Gastroenterol 2011;49:350-356. 